\title{
RANK TWO VECTOR BUNDLES WITH CANONICAL DETERMINANT
}

\author{
MONTSERRAT TEIXIDOR I BIGAS
}

\begin{abstract}
Denote by $B_{2, K}^{k}$ the locus of vector bundles of rank two that have canonical determinant and at least $k$ sections. We show that for a generic curve of genus $g, B_{2, K}^{k}$ is non-empty and has a component of the expected dimension if $g$ is sufficiently large.
\end{abstract}

\section{INTRODUCTION}

Denote by $U(r, d)$ the moduli space of stable vector bundles of rank $r$ and degree $d$ on a fixed curve $C$ of genus $g$. The Brill-Noether loci $B_{r, d}^{k}$ are defined as the subsets of $U(r, d)$ consisting of vector bundles with $k$ sections (note that we use $k$ rather than $k-1$ in the notation). These can be given a locally determinantal scheme structure. As such the expected dimension of $B_{r, d}^{k}$ for a generic curve is given by the Brill-Noether number $\rho=r^{2}(g-1)+1-k(k-d+r(g-1))$ and the expected singular locus is $B_{d, r}^{k+1}$. The case $r=1$ is classical and all the expected results hold for the generic curve. For higher rank though, several cases are known where $\rho$ is positive and the locus is empty (cf [BGN]) or $\rho$ is negative and the locus is non-empty (use $\mathrm{Me}$ ) or the singular locus is larger than expected (see [T1]). We shall add to the list of abnormalities by showing that $B_{2,2 g-2}^{k}$ is sometimes of dimension larger than expected for the generic curve and therefore reducible (see also $[\mathrm{BF}]$ ).

Denote now by $U(r, L)$ the moduli space of stable vector bundles of rank $r$ and determinant $L$. Define similarly $B_{r, L}^{k}$ as the subset of $U(r, L)$ consisting of vector bundles with $k$ sections. For $k>r$ (and $d \leq 2 g-2$ ), one expects that the geometry of $B_{r, L}^{k}$ will very much depend on the position of $L$ itself as a point of various $B_{1, d}^{j}$. The case $L=O$ is not very interesting as a stable vector bundle of degree zero cannot have sections. It is then natural to look at the special case when $L=K$ is the canonical bundle. When $r=2$, this locus has a natural structure as a symmetric degeneracy locus (see $\underline{\mathrm{Mu}}, \underline{\mathrm{H}}, \underline{\mathrm{Mk}} \mathbf{\mathrm { B }}, \underline{\mathrm{BF}}, \underline{\mathrm{Mk}}]$ ). Using this description, the expected dimension of $B_{2, K}^{k}$ is given by $\rho_{K}^{k}=3 g-3-\left(\begin{array}{c}k+1 \\ 2\end{array}\right)$. By its definition, this is the minimum dimension of any component of these loci.

One can again ask the question ( $\mathrm{Mk} 1$ ) of whether the locus is non-empty for $\rho_{K}^{k}>0$ and of dimension $\rho_{K}^{k}$ and empty for $\rho_{K}^{k}<0$. It was conjectured in $[\mathrm{BF}]$ that this would be the case. Some evidence for this conjecture using curves of small genus was provided in $[\underline{R}$. The main purpose of this paper is to partly prove these statements.

We want to mention that the study of the loci $B_{2, K}^{k}$, apart from its intrinsic interest, seem to contribute a lot to our knowledge of the geometry of the curves. Mukai for example showed in Mk2 that if $C$ is a curve of genus eleven, then $W_{2, K}^{7}$ 
is a $K 3$ surface containing the curve. Something can be said also for curves of higher genus.

Moreover, as $B_{2, K}^{k} \subset B_{2,2 g-2}^{k}$, a study of $B_{2, K}^{k}$ will sometimes provide some information on $B_{2,2 g-2}^{k}$ (see 1.2).

Our main result is the following

1.1. Theorem Let $C$ be a generic curve of genus $g$. If $k=2 k_{1}$ is even, then $B_{2, K}^{k}$ is non-empty when

$$
g \geq k_{1}^{2} \text { if } k_{1}>2, g \geq 5 \text { if } k_{1}=2, g \geq 3 \text { if } k_{1}=1 .
$$

Moreover, under these conditions, it has a component of the right dimension $\rho_{K}^{k}$.

If $k=2 k_{1}+1$ is odd, then $B_{2, K}^{k}$ is non-empty when $g \geq\left(k_{1}\right)^{2}+k_{1}+1$. Moreover, under these conditions, it has a component of the right dimension.

1.2. Corollary If $C$ is a generic curve of genus $g,\left(k_{1}\right)^{2} \leq g<2\left(k_{1}\right)^{2}-k_{1}$, then $B_{2,2 g-2}^{2 k_{1}}$ is non-empty and has a component of dimension larger than expected.

If $C$ is a generic curve of genus $g,\left(k_{1}\right)^{2}+k_{1}+1 \leq g<2\left(k_{1}\right)^{2}+k_{1}$, then $B_{2,2 g-2}^{2 k_{1}+1}$ is non-empty and has a component of dimension larger than expected.

The author is a member of the EAGER group "Vector bundles on Algebraic Curves"

\section{Proof of EXISTEnCE}

We start by recalling some definitions and results that we shall later need.

2.1. Chain of elliptic curves Let $C_{i}, i=1 \ldots g$ be elliptic curves. Let $P_{i}, Q_{i}$ be generic points on $C_{i}$. Take $C$ to be the curve obtained by identifying $Q_{i}$ and $P_{i+1}$.

In order to give linear series on elliptic curves it will be useful to have the following

2.2. Remark One can define a subspace of dimension $k$ of sections of a fixed line bundle $L$ of degree $d$ by specifying the $k$ distinct (minimum) desired vanishings of a basis of the subspace at two different points $P, Q$ so that the sum of the corresponding vanishings at $P, Q$ is $d-1$. In the case when $L=O(a P+(d-a) Q)$, two of the vanishings could be chosen to be $a, d-a$ (adding up to $d$ rather than $d-1)$. These are the only two vanishings that can add to $d$ if $P, Q$ are generic.

Proof. Assume given a line bundle of degree $d$ on an elliptic curve $C$ and two points $P, Q$ on $C$. If $L$ is general, there is no section $s$ of $L$ such that the orders of vanishing of $s$ at $P$ and $Q$ add up to $d$. If such a section exists, then $L=O(a P+(d-a) Q)$ for some $a \leq d$. Moreover, if $P, Q$ are generic (i.e. they don't differ in a torsion point of order at most $d$ ) such an $a$ is unique.

On the other hand, there is always a section (defined up to a constant) whose orders of vanishing at $P, Q$ are at least given numbers $a, b$ satisfying $a+b=d-1$. If $L$ is generic, the vanishings of this section at $P, Q$ are precisely $a, b$ respectively. If $L=O(a P+(d-a) Q)$, then the vanishings are $a, d-a=b+1$ respectively.

Hence, our statement is proved.

To simplify our task, we shall always list the vanishing $u_{j}$ at $P$ in increasing order and the vanishing $v_{j}$ at $Q$ in decreasing order.

A similar argument can be used to define sections of a given rank two vector bundle. Now every vanishing at $P, Q$ may appear twice. 
On a reducible curve, the equivalent of a linear series is a limit linear series introduced by Eisenbud and Harris (cf $[\mathrm{EH}$ ) for the rank one case and later generalised to arbitrary rank in [1]. For the convenience of the reader, we reproduce the definition here:

2.3. Limit linear series of rank $r$ dimension $k$ and degree $d$ on a chain of $M$ (not necessarily elliptic) curves consists of data I, II below for which data III,IV exist satisfying conditions a-c.

I) For every component $C_{i}$, a vector bundle $E_{i}$ of rank $r$ and degree $d_{i}$ and a $k$-dimensional space of sections of $E_{i}$.

II) For every node obtained by gluing $Q_{i}$ and $P_{i+1}$ an isomorphism of the projectivisations of the fibers $\left(E_{i}\right)_{Q_{i}}$ and $\left(E_{i+1}\right)_{P_{i+1}}$.

III) A positive integer a.

$I V)$ For every node obtained by gluing $Q_{i}$ and $P_{i+1}$, basis $s_{Q_{i}}^{t}, s_{P_{i+1}}^{t}, t=1 \ldots k$ of the vector spaces $V_{i}$ and $V_{i+1}$.

Subject to the conditions

a) $\sum_{i=1}^{M} d_{i}-r(M-1) a=d$

b) The orders of vanishing at $Q_{i}, P_{i+1}$ of the sections of the chosen basis satisfy $s_{Q_{i}}^{t}+s_{P_{i+1}}^{t} \geq a$

c) Sections of the vector bundles $E_{i}\left(-a P_{i}\right), E_{i}\left(-a Q_{i}\right)$ are completely determined by their value at the nodes.

Example When $r=1$ (cf $[\mathrm{EH}]$ ) the gluing in II is irrelevant. One can take $d_{i}=d$ and then $a=d$ too. Conditions a) and c) are automatically satisfied and one needs only check condition b).

For instance, the limit of the canonical series on a curve as in 2.1 is the unique linear series of rank one dimension $g$ and degree $2 g-2$. One can check that this limit is given as follows:

$$
E_{i}=O\left((2 g-2 i) Q_{i}+(2 i-2) P_{i}\right) .
$$

The space of sections is the unique subspace of $H^{0}\left(O\left((2 g-2 i) Q_{i}+(2 i-2) P_{i}\right)\right)$ whose vanishing $u_{j}^{i}, v_{j}^{i}$ at $P_{i}, Q_{i}$ are given by,

$u_{e}^{i}=i-3+e, e<i, u_{k}^{i}=i-2+e, e \geq i, v_{e}^{i}=2 g-i-e, e \leq i, v_{e}^{i}=2 g-i-e-1, e>i$ (use Remark 2.2)

Proof. We now turn to the proof of 1.1 The main fact that we use in the proof is the following: As $B_{2, K}^{k}$ is defined as the pull-back of a symmetric degeneracy locus, its dimension at every point is at least $\rho_{K}^{k}$. Even better, given a family of curves $\mathbf{C} \rightarrow B$ one can define similarly $\mathbf{B}_{2, K}^{k}$ parametrising pairs consisting of a curve in the family and a vector bundle of rank two with canonical determinant and at least $k$ sections. Then, $\operatorname{dim}\left(\mathbf{B}_{2, K}^{k}\right) \geq \operatorname{dim}(B)+\rho_{K}^{k}$ at every point. If we can find a curve $C_{0}$ in such a family for which $\operatorname{dim}\left(B_{2, K}^{k}\right)=\rho_{K}^{k}$, then the generic fiber of the map $\mathbf{B}_{2, K}^{k} \rightarrow B$ is non-empty and has dimension $\rho_{K}^{k}$. This is what we shall do with a curve $C_{0}$ as in 2.1

We first prove existence in the case of even $k=2 k_{1}$ and under the assumption $g \geq\left(k_{1}\right)^{2}\left(g>\left(k_{1}\right)^{2}\right.$ if $\left.k_{1} \leq 2\right)$.

We must give a family of limit linear series on a curve as in 2.1 of rank two dimension $k$ and canonical determinant. We need to show that the dimension of this family is $3 g-3-\left(\begin{array}{c}k+1 \\ 2\end{array}\right)$ and that this family is not part of a larger family of linear series with canonical determinant. 
Consider the first $\left(k_{1}\right)^{2}$ components $C_{i}$. Write

$$
i=a^{2}+2 c+\epsilon, 0 \leq a \leq k_{1}-1,0 \leq c \leq a-1, \epsilon=1,2, \text { or } c=a, \epsilon=1
$$

Take the vector bundle to be of the form

$$
O((g-i-c+a) Q+(c-a+i-1) P) \oplus O((g-i-a+c) Q+(a-c+i-1) P)
$$

Note that the determinant of this vector bundle is $O((2 g-2 i) Q+2(i-1) P)$ corresponding to the canonical bundle. Take the space of sections whose vanishing $u_{j}^{i}, v_{j}^{i}$ at $P_{i}, Q_{i}$ are given respectively by

$$
\begin{gathered}
u_{2 e-1}^{i}=u_{2 e}^{i}=i+e-a-3, v_{2 e-1}^{i}=v_{2 e}^{i}=g-i-e+a+1, e \leq c \\
u_{2 c+1}^{i}=i+c-a-1=1, u_{2 c+1}^{i}=i+c-a-2 \text { if } \epsilon=2 \\
u_{2 c+2}^{i}=i+c-a-1 \\
v_{2 c+1}^{i}=g-i-c+a, v_{2 c+2}^{i}=g-i-c+a-1 \text { if } \epsilon=1, v_{2 c+2}^{i}=g-i-c+a \text { if } \epsilon=2 \\
u_{2 e-1}^{i}=u_{2 e}^{i}=i+e-a-2, v_{2 e-1}^{i}=v_{2 e}^{i}=g-i-e+a, c+1<e \leq a \\
i \neq a^{2}+2 a+1, u_{2 a+1}^{i}=i-c+a-1 \text { if } \epsilon=1, u_{2 a+1}^{i}=i-c+a-2 \text { if } \epsilon=2 \\
u_{2 a+2}^{i}=i+a-c-1 \\
v_{2 a+1}^{i}=g-i+c-a, v_{2 a+2}^{i}=g-i-a+c-1 \text { if } \epsilon=1, v_{2 c+2}^{i}=g-i-a+c \text { if } \epsilon=2 \\
i=a^{2}+2 a+1, u_{2 a+1}^{i}=u_{2 a+2}^{i}=i-1, v_{2 a+1}^{i}=v_{2 a+2}^{i}=g-i \\
u_{2 e-1}^{i}=u_{2 e}^{i}=i+e-2, v_{2 e-1}^{i}=v_{2 e}^{i}=g-i-e, e=a+2 \ldots k
\end{gathered}
$$

We use Remark 2.2 to show that such a space of sections of the given vector bundle exists (and is unique satisfying the conditions). We need to show that the vanishing $u_{\alpha}, v_{\alpha}$ add up to $g-2$ except for two values $\bar{u}_{\alpha}, \bar{v}_{\alpha}, \hat{u}_{\alpha}, \hat{v}_{\alpha}$ that add up to $g-1$ and then

For $\epsilon=1$,

$$
E_{i}=O\left(\bar{u}_{\alpha} P_{i}+\bar{v}_{\alpha} Q_{i}\right) \oplus O\left(\hat{u}_{\alpha} P_{i}+\hat{v}_{\alpha} Q_{i}\right)
$$

$$
u_{\alpha}^{i}+v_{\alpha}^{i}=g-2, \alpha \neq 2 c+1,2 a+1 u_{\alpha}^{i}+v_{\alpha}^{i}=g-1, \alpha=2 c+1,2 a+1
$$

and

Similarly, for $\epsilon=2$

$$
E_{i}=O\left(u_{2 c+1}^{i} P_{i}+v_{2 c+1}^{i} Q_{i}\right) \oplus O\left(u_{2 a+1}^{i} P_{i}+v_{2 a+1}^{i} Q_{i}\right)
$$

$$
\begin{gathered}
u_{\alpha}^{i}+v_{\alpha}^{i}=g-2, \alpha \neq 2 c+2,2 a+2 u_{\alpha}^{i}+v_{\alpha}^{i}=g-1, \alpha=2 c+2,2 a+2 \\
E_{i}=O\left(u_{2 c+2}^{i} P_{i}+v_{2 c+2}^{i} Q_{i}\right) \oplus O\left(u_{2 a+2}^{i} P_{i}+v_{2 a+2}^{i} Q_{i}\right)
\end{gathered}
$$

Finally, for $c=a, \epsilon=1$ (and hence $i=a^{2}+2 a+1=(a+1)^{2}$ )

$$
\begin{gathered}
u_{\alpha}^{i}+v_{\alpha}^{i}=g-2, \alpha \neq 2 a+1,2 a+2 u_{\alpha}^{i}+v_{\alpha}^{i}=g-1, \alpha=2 a+1,2 a+2 \\
E_{i}=O\left(u_{2 a+1}^{i} P_{i}+v_{2 a+1}^{i} Q_{i}\right) \oplus O\left(u_{2 a+2}^{i} P_{i}+v_{2 a+2}^{i} Q_{i}\right)
\end{gathered}
$$

On the curves $C_{i}, i>k^{2}$, take direct sum of an arbitrary line bundle of degree $g-1$ and its complement with respect to the limit canonical series, namely

$$
L_{i} \oplus L_{i}^{\prime}, \quad \text { such that } L_{i} \otimes L_{i}^{\prime}=O\left(2(g-i) Q_{i}+2(i-1) P_{i}\right)
$$

There is a well determined subspace of dimension $k$ of sections of $E_{i}$ with given vanishings $u_{j}^{i}$ at $P_{i}$ and $v_{j}^{i}=g-2-u_{j}^{i}$ at $Q_{i}$. Take

$$
u_{2 e-1}^{i}=u_{2 e}^{i}=i+e-k_{1}-2, v_{2 e-1}^{i}=v_{2 e}^{i}=g-i+k_{1}-e, i=\left(k_{1}\right)^{2}+1 \ldots g-1, e=1 \ldots k_{1}
$$

Take then the gluing at the nodes so that the section with vanishing $v_{j}^{i-1}$ glues with the section with vanishing $u_{j}^{i}$. 
One can check that these spaces satisfy the conditions on the degree and vanishing of a limit linear series taking $a=g-1$ : As every vector bundle is a direct sum of two line bundles of degree $g-1=a$, the condition on the sums of degrees is satisfied and any section of $E_{i}\left(-a P_{i}\right)$ or $E_{i}\left(-a Q_{i}\right)$ is determined by its value at the node. Also, using the explicit expression for the $u_{j}^{i}, v_{j}^{i}$, one can check that $v_{j}^{i-1}+u_{j}^{i}=a$ for all pairs $i, j$.

The given family of linear series is not part of a larger family of linear series of dimension $k$ with fixed determinant $K$ : the two ways in which one could make the vector bundle more general are either by taking on some of the first $\left(k_{1}\right)^{2}$ components a generic sum of two line bundles (with product equal to the restriction of the canonical) or by taking more general gluings. In either case, the vanishings $v_{j}^{i}$ at the corresponding component $C_{i}$ will decrease. The values of the $v_{j}^{g-1}$ at $C_{g-1}$ were

$$
k, k, k-1, k-1, \ldots 2,2,1,1
$$

If they were smaller, it would no longer be possible to glue this linear series with two generic line bundles on $C_{g}$ and preserve the sections.

We now count the dimension of the family obtained in this way.

The gluing of $C_{a^{2}+2 c}$ with $C_{a^{2}+2 c+1}$ is free because for every vanishing there is a two dimensional space of sections with this vanishing Therefore, these gluings depend on four parameters. On the other hand, the gluing of $C_{a^{2}+2 c+1}$ with $C_{a^{2}+2 c+2}$ must glue two given directions to each other. Hence, it depends on only two parameters. The gluing between $C_{a^{2}+2 a}$ and $C_{a^{2}+2 a+1}$ is again free. So, between $C_{a^{2}}$ and $C_{(a+1)^{2}}$ there are $a+1$ gluings depending on four parameters and $a$ gluings depending on two parameters.

The remaining $g-\left(k_{1}\right)^{2}$ gluings are free and depend on four parameters each. The total contribution of the gluings is

$4\left[\left(2+3+4+\ldots+k_{1}\right)+g-\left(k_{1}\right)^{2}\right]+2\left(1+2+\ldots+\left(k_{1}-1\right)\right)=4 g-\left(k_{1}\right)^{2}+k_{1}-4$

The first $\left(k_{1}\right)^{2}$ vector bundles are well determined while the remaining $g-\left(k_{1}\right)^{2}$ depend on the choice of a line bundle of degree $g-1$ on the elliptic curve (the second line bundle being its conjugate with respect to the line bundle giving the canonical linear series). As the jacobian of an elliptic curve is one dimensional, this contributes $g-\left(k_{1}\right)^{2}$ parameters in all.

We now need to compute the (negative) contribution of the endomorphisms. The direct sum of two different line bundles has a two dimensional family of endomorphisms. The direct sum of two identical line bundles has a four dimensional family of endomorphisms. The vector bundles $E_{1}, E_{4}, E_{9}, \ldots, E_{\left(k_{1}\right)^{2}}$ are of the latter type, all of the remaining ones of the former type.

Hence, the dimension of the family is

$$
4 g-\left(k_{1}\right)^{2}+k_{1}-4+g-k_{1}^{2}-4 k_{1}-2\left(g-k_{1}\right)+1
$$

where the last +1 comes from the fact that the resulting vector bundle is stable (see below) and therefore has a one dimensional family of endomorphisms. The number above equals

$$
3 g-2\left(k_{1}\right)^{2}-k_{1}-3=3 g-3-\left(\begin{array}{c}
k+1 \\
2
\end{array}\right)=\rho_{K}^{k}
$$

as promised.

It remains to show that the vector bundle that we obtain in this way is stable. As the restriction to each of the elliptic components is semistable, using [T2 or [T3], the resulting vector bundle is semistable. We need to check that it is actually 
stable. It suffices then to show that the line subbundles that make the restriction to the elliptic components strictly semistable, don't glue with each other. If $g \geq$ $k_{1}^{2}+1$, there are precisely two subline bundles of $E_{g}$ that contradict stability. If $k_{1}=2, g=5$, there are two subline bundles on the chain consisting of the first four elliptic curves that contradict stability. But by the genericity of the gluing between $C_{4}$ and $C_{5}$, these don't glue together. If $k_{1} \geq 3$, no line subbundles on the first $k_{1}^{2}$ components contradict stability. This completes the proof of stability for the resulting bundle.

We now turn to the case of an odd number of sections $k=2 k_{1}+1$

Proof. On the first $k_{1}$ elliptic components, one takes the same vector bundles and spaces of sections with just one more section that in the case $k=2 k_{1}$. This new section vanishes with multiplicities

$$
u_{2 k_{1}+1}^{i}=i+k_{1}-1, v_{2 k_{1}+1}^{i}=g-i-k_{1}-1
$$

so

Hence

$$
u_{2 k_{1}+1}^{i}+v_{2 k_{1}+1}^{i}=g-2
$$

$$
u_{2 k_{1}+1}^{i}=u_{2 k_{1}}^{i}+1, v_{2 k_{1}+1}^{i}=v_{2 k_{1}}^{i}-1, i=1 \ldots\left(k_{1}\right)^{2}
$$

On the curves $C_{i}, i=k_{1}^{2}+m, 1 \leq m \leq k_{1}$ we take the vector bundle to be

$O\left(\left(g-i-m+k_{1}+1\right) Q+\left(i+m-k_{1}-2\right) P\right) \oplus O\left(\left(g-\left(k_{1}\right)^{2}-k_{1}-1\right) Q+\left(\left(k_{1}\right)^{2}+k_{1}\right) P\right)$

The vanishing $u_{j}^{i}, v_{j}^{i}$ of the sections of the linear series at the points $P_{i}, Q_{i}$ in the curve $C_{i}, i=\left(k_{1}\right)^{2}+m, 1 \leq m \leq k_{1}$ are given by

$$
\begin{gathered}
u_{2 e-1}=i+e-k_{1}-3, e<m, u_{2 e-1}=i+e-k_{1}-2, m \leq e \leq k_{1}, u_{2 k_{1}+1}=\left(k_{1}\right)^{2}+k_{1} \\
u_{2 e}=i+e-k_{1}-2, e \leq k_{1} \\
v_{2 e-1}=g-i-e+k_{1}+1, e \leq m, v_{2 e-1}=g-i-e+k_{1}, m<e \leq k_{1}, \\
v_{2 k_{1}+1}=g-1-\left(k_{1}\right)^{2}-k, v_{2 e}=g-i-e+k_{1}, e \leq k_{1}
\end{gathered}
$$

On the curve $C_{\left(k_{1}\right)^{2}+k_{1}+1}$, take the unique indecomposable vector bundle of rank two and degree $2 g-2$ which has one section vanishing with multiplicity $g-1-$ $\left(k_{1}\right)^{2}-k_{1}$ at $\mathrm{Q}$ and $\left(k_{1}\right)^{2}+k_{1}$ at $\mathrm{P}$. On the remaining curves, take again a direct sum of two line bundles each of degree $g-1$ and whose product is the restriction of the canonical to this component.

In order to compute the dimension of this family, note that on the first $\left(k_{1}\right)^{2}$ components, the counts are the same as in the even number of sections case. The gluing between the curve $C_{\left(k_{1}\right)^{2}}$ and $C_{\left(k_{1}\right)^{2}+1}$ is free and therefore depends on four parameters. The gluings between $C_{\left(k_{1}\right)^{2}+i}$ and $C_{\left(k_{1}\right)^{2}+i+1}$ must glue together the direction corresponding to the vanishings $v_{2 k_{1}+1}^{\left(k_{1}\right)^{2}+i}$ and $u_{2 k_{1}+1}^{\left(k_{1}\right)^{2}+i+1}$ Hence, they depend on three parameters. All vector bundles $E_{k_{1}^{2}+i}, i=1 \ldots k_{1}+1$ have a two dimensional family of endomorphisms. Computing as before, we find that the dimension of the family is $\rho_{K}^{k}$

Again, the vector bundle obtained in this form is stable for $k_{1} \geq 2$ and for $k_{1}=1, g \geq 4$. For $k_{1}=1, g=3$ one can take as the vector bundle $E$ on any non-singular non-hyperelliptic curve the dual of the kernel of the evaluation map defined by

$$
0 \rightarrow E^{*} \rightarrow H^{0}(K) \otimes O_{C} \rightarrow K \rightarrow 0
$$

This is known to be stable $\mathrm{P}, \mathrm{R}$. 


\section{REFERENCES}

[BF] A.Bertram, B.Feinberg On stable rank two bundles with canonical determinant and many sections in "Algebraic Geometry, papers for Europroj Conferences in Catania and Barcelona" editor P.Newstead 1998, 259-269.

[BGN] 1.Brambila-Paz, I.Grzegorczyk, P.Newstead Geography of Brill-Noether loci for small slope, J.Algebraic Geometry 6(1997), 645-669.

[EH] D.Eisenbud, J.Harris Limit linear series, basic theory, Invent.Math.(1986), 637-662.

$[\mathrm{H}]$ J.Harris Theta characteristics on singular curves, Trans.AMS.271, N2, 1982, 611-638.

[Me] V.Mercat On extensions of line bundles and a Theorem of Teixidor, Man.Math.98, 1999, 75-85.

[Mk1] S.Mukai, Curves and Brill-Noether Theory in Current Topics in Algebraic Geometry, Math.Sci. Res Inst.Publ 28, Cambridge University Press 1995, 145-158.

[Mk2] S.Mukai, Curves and K3 surfaces of genus eleven in Moduli of vector bundles, M.Maruyama (editor), Marcel Dekker, New York (1996), 180-197.

[Mk3] S.Mukai, Non-abelian Brill-Noether Theory and Fano 3-folds

[Mu] D.Mumford Theta characteristics on an algebraic curve, Ann.Sci.Ec.Norm.Sup.4(1971), 181-192.

[O,P] W.Oxbury, C.Pauly Subvarieties of Heisenberg invariant quartics and $S U_{C}(2)$ for a curve of genus four. Preprint.

$[\mathrm{O}, \mathrm{P}, \mathrm{P}]$ W.Oxbury, C.Pauly, E.Previato Subvarieties of $S U_{C}(2)$ and $2 \theta$ divisors in the Jacobian Preprint

$[\mathrm{P}, \mathrm{R}]$ R.Paranjape, S.Ramanan, On the canonical ring of a curve, Algebraic Geometry and Commutative Algebra in honour of M.Nagata (1987) 503-516.

[R] A.Rayfield Brill-Noether loci for rank 2 Vector bundles Preprint.

[T1] M.Teixidor Brill-Noether Theory for stable vector bundles, Duke Mathematical Journal 62 N2 (1991), 385-400.

[T2] M.Teixidor Moduli spaces of semistable vector bundles on tree-like curves, Math.Ann.290 (1991), 341-348.

[T3] M.Teixidor Moduli spaces of vector bundles on reducible curves, Amer.J of Math. 219(1995), 477-484.

Mathematics Department, Tufts University, Medford Ma02155, USA 